\title{
A comparison of patient testimonials on YouTube of the most common orthodontic treatment modalities: braces, in-office aligners, and direct-to-consumer aligners
}

Riley Hunsaker

vCU Orthodontics

Follow this and additional works at: https://scholarscompass.vcu.edu/etd

(C) The Author

\section{Downloaded from}

https://scholarscompass.vcu.edu/etd/6197

This Thesis is brought to you for free and open access by the Graduate School at VCU Scholars Compass. It has been accepted for inclusion in Theses and Dissertations by an authorized administrator of VCU Scholars Compass.

For more information, please contact libcompass@vcu.edu. 
(C) Riley Jeff Hunsaker, DDS

All Rights Reserved 


\section{A comparison of patient testimonials on YouTube of the most common orthodontic treatment modalities: braces, in-office aligners, and direct-to-consumer aligners}

A thesis submitted in partial fulfillment of the requirements for the degree of Master of Science in Dentistry at Virginia Commonwealth University.

\section{By}

Riley J. Hunsaker, D.D.S.

B.A., Business Management, Brigham Young University, 2014

D.D.S., University of Utah School of Dentistry, 2018

Thesis Advisor: Bhavna Shroff, D.D.S., M.Dent.Sc., M.P.A.

Department of Orthodontics, Program Director

Virginia Commonwealth University

Richmond, Virginia

April 2020 


\section{Acknowledgements}

I am beyond grateful for my orthodontic education at Virginia Commonwealth University and I would like to thank Drs. Steven Lindauer, Bhavna Shroff, and Eser Tüfekçi for making this dream a reality. I am grateful to Dr. Shroff, for her support and encouragement on this project and throughout residency. Thank you Dr. Lindauer for your leadership and teaching, and thank you Dr.Tüfekçi for imparting your knowledge and love onto each of us. I would also like to thank Dr. Carrico for her excellent contributions. Lastly, I would like to thank my wife Kenzie, for her unwavering support, love, and patience throughout this journey, as well as our children, Hansen and Hazel, for providing endless joy and laughter. 


\section{Table of Contents}

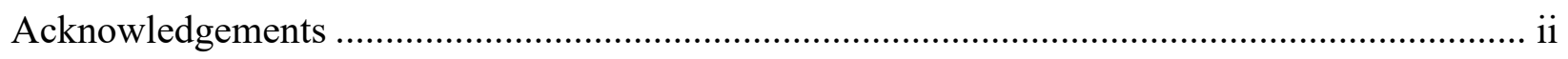

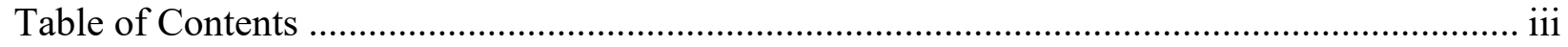

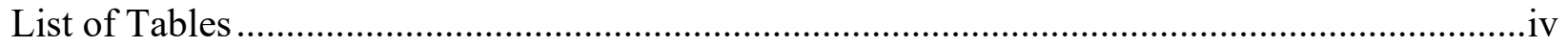

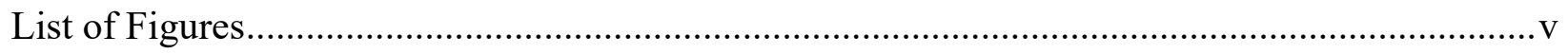

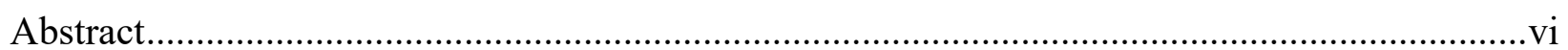

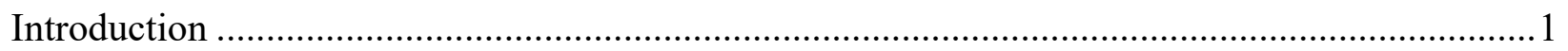

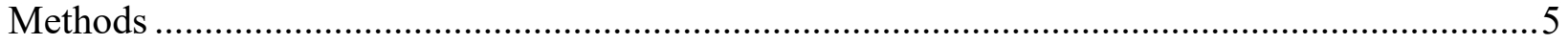

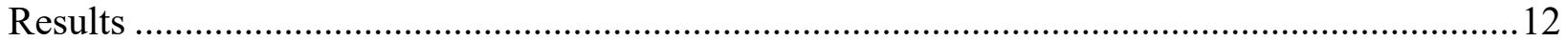

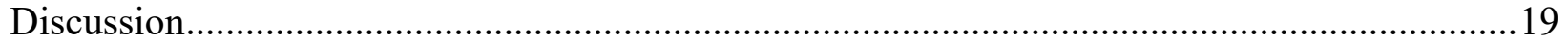

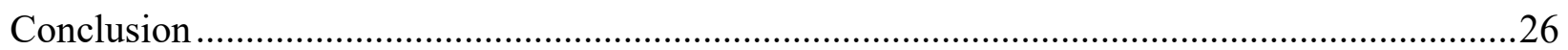

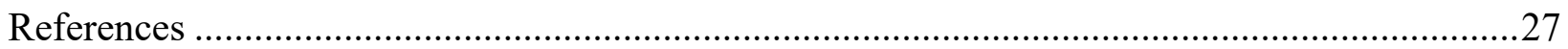




\section{List of Tables}

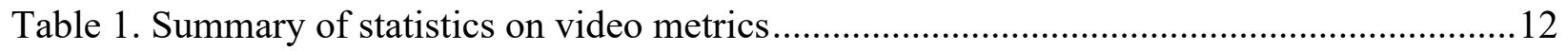

Table 2. Association between video metrics and ICS ............................................................ 14

Table 3. Associations between video metrics across treatment type...................................... 16

Table 4. Relationship between treatment modality and comment sentiment ............................ 18 


\section{List of Figures}

Figure 1. Video selection process for braces $(\mathrm{B})$............................................................

Figure 2. Video selection process for in-office aligners (IOA) .............................................. 10

Figure 3. Video selection process for direct-to-consumer aligners (DTCA) ............................11

Figure 4. Most common words found in positive comments among all three groups .................31

Figure 5. Most common words found in negative comments among all three groups .................32

Figure 6. Most common words found in negative comments from B videos ...........................33

Figure 7. Most common words found in negative comments from IOA videos .........................34

Figure 8. Most common words found in negative comments from DTCA videos ......................35 


\begin{abstract}
A COMPARISON OF PATIENT TESTIMONIALS ON YOUTUBE OF THE MOST COMMON ORTHODONTIC TREATMENT MODALITIES: BRACES, IN-OFFICE ALIGNERS, AND DIRECT-TO-CONSUMER ALIGNERS
\end{abstract}

\author{
By: Riley J. Hunsaker, D.D.S. \\ A thesis submitted in partial fulfillment of the requirements for the degree of Master of Science \\ in Dentistry at Virginia Commonwealth University \\ Virginia Commonwealth University, 2020 \\ Thesis Advisor: Bhavna Shroff, D.D.S., M.Dent.Sc., M.P.A. \\ Department of Orthodontics, Program Director
}

Introduction: The objectives of this research was to investigate and compare the educational value of the most popular YouTube orthodontic patient testimonials between braces (B), inoffice aligners (IOA), and direct-to-consumer aligners (DTCA), and to classify the emotional response of the viewers through a sentiment analysis of the video comments.

Methods: Three different phrases relevant to B, IOA, and DTCA were searched on YouTube. The 20 most popular patient testimonial videos that met the criteria for each group were selected, for a total of 60 videos. Using the YouTube API for each video, 13 video metrics were extracted, an information completeness score (ICS) was assigned, and an analysis of the video comments was performed using sentiment analysis software.

Results: The 60 videos included in this study were viewed 34,384,786 times by internet users. Braces videos have significantly more likes, comments, and a higher viewer interaction score than the IOA and DTCA videos. IOA videos had a higher median ICS than B and DTCA videos. Of the 5149 video comments with polarity, $53.6 \%$ were positive and $46.4 \%$ were negative 
$(\mathrm{P}<0.0001)$. There was not a significant association between the treatment modality and positive or negative comments $(\mathrm{P}=0.5679)$.

Conclusions: There is high user engagement on YouTube with orthodontic patient testimonials. YouTube users interact with braces patient testimonials the most. YouTube viewers' comments on orthodontic patient testimonials express more positive sentiment than negative sentiment. There is no significant difference in positive and negative sentiment between the video comments for the three different treatment modalities. 


\section{Introduction}

Personal health decision-making is a complex process that involves the consumption of information both in statistical and in narrative forms. While the statistical form of information is often derived from methodical research with substantiated claims, the narrative form is the primary method of human interaction and consequently plays a critical role in healthcare.

Providers can improve their care by better understanding their patient's experiences, and patients often rely on the narratives of others to make decisions for themselves. Researchers have often debated which of these two forms of healthcare communication is most persuasive.

Social media is a platform in which narrative communication has a very prominent role. Publicly available information from social media sites continues to be utilized by researchers to better understand the patient experience. Patient testimonials, a form of narrative communication, are prevalent throughout the rapidly expanding social media universe and fall into a category now known as health video blogs, or health vlogs, with the term "vlogger" identifying individuals who publish short videos to their vlogs. The anecdotal information found in testimonials often has the potential to be more compelling than statistical and evidence-based information because it is concrete and easier to relate to for the average healthcare consumer. ${ }^{1}$

Facebook is the most popular social media platform, but YouTube, a popular videosharing website and a Google subsidiary, is ranked $2^{\text {nd }}$ worldwide and in the United States for internet traffic, boasting over 2 billion monthly active users. ${ }^{2}$ YouTube allows its users to view, upload, rate, and comment on videos. While most content on YouTube is uploaded by individuals, many corporations contribute their own videos as well as sponsor others to upload videos. YouTube's impact on society has continued to increase since its inception in 2005 . There 
are now more than 500 hours of video content uploaded to the platform every minute. The site will most likely continue in popularity as video content is expected to make up $82 \%$ of all internet traffic by $2021 .^{3}$

Even though we live in an age of digital information, misinformation is just as prevalent as correct information and has the ability to influence the average internet user to accept, reject, or question a certain treatment. For example, unproven stem cell treatments are marketed heavily to consumers on YouTube throughout the world in the form of patient testimonials, and the amount of misinformation contained in these videos has resulted in physical, financial, and emotional harm to patients. ${ }^{4}$ The majority of research conducted on the dissemination of health information on YouTube has led to similar conclusions; professional institutions must work to produce more peer-reviewed content to improve the availability of credible health information on internet platforms, and these institutions and providers must play an active role in directing patients toward these alternative high-quality information sources. ${ }^{5-7}$

Different methods have been employed to assess the content, quality, and popularity of YouTube videos. The DISCERN tool has been commonly used to evaluate the reliability and quality of online written health information and treatment choices but falls short in assessing video-based information from patient testimonials. The Oxford Textbook of Global Public Health has delineated five criteria, which are easily identifiable in video format, that have been shown to epitomize the overall "completeness of information" of a patient testimonial and to attract more attention on social media. ${ }^{8,9}$ Sentiment analysis, often referred to as opinion mining, is a method primarily used to extract and characterize subjective information. Sentiment analyses on Twitter have been used frequently to study a broad range of fields, including political election predictions, box office reactions, stock market indicators, and even the orthodontic patient 
experience. ${ }^{10}$ The premise behind social media opinion mining is to analyze consumer feedback and to predict future consumer behavior. ${ }^{11}$ YouTube is a vast information source that continues to be investigated in the medical and dental fields.

The word "orthodontics" was searched on Google 100\% more times in 2017 than in 2016, totaling 60 million searches. ${ }^{12}$ The public frequently uses YouTube to engage in conversation and obtain information about orthodontic treatment, and the most common video category on YouTube regarding orthodontics is that of patient testimonials. ${ }^{13,14}$ As many individuals continue to seek orthodontic treatment, educating patients on the topic is more important now than ever. The specialty of orthodontics is seeing a large expansion of potential patients as orthodontic companies flood the internet, social media, and television outlets with advertisements about improving one's smile. The current literature on YouTube and orthodontics is limited and most studies have only focused on identifying the educational value and accuracy of videos relating to a specific orthodontic topic, but have not focused on patient testimonials. ${ }^{15-}$ ${ }^{17}$ However, a recent YouTube study focused solely on the Invisalign ${ }^{\circledR}$ patient experience. ${ }^{9}$ The popularity of clear aligner therapy has grown drastically over the last 10-15 years and providers should have a detailed understanding of the benefits and drawbacks from the patients' perspectives of both fixed appliances (braces) and clear aligner therapy.

Regardless of the treatment modality, orthodontic treatment satisfaction has been shown to be high, with a tendency to be consistently higher than general dental treatment. ${ }^{18,19}$ Many studies have identified variables influencing patient satisfaction with traditional fixed appliances, including the gender and age of patient, the duration of treatment, and the attention and care received during treatment. ${ }^{20,21}$ Similar studies have been carried out with clear aligner therapy showing that patients are highly satisfied during treatment. ${ }^{22} \mathrm{~A}$ Twitter analysis comparing the 
overall sentiment of comments pertaining to braces and Invisalign ${ }^{\circledR}$ demonstrated no significant difference between both groups. ${ }^{10}$ One study assessing the treatment satisfaction and periodontal health of traditional braces patients versus clear aligner patients demonstrated that the clear aligner group had a greater periodontal health and higher treatment satisfaction than those treated with braces. ${ }^{23}$ With regard to treatment effectiveness, both braces and clear aligners have been shown to be effective in treating malocclusion, with each of them possessing different strengths and weaknesses. ${ }^{24}$

Direct-to-consumer (DTC) offerings have become more available in general retail and in healthcare (genetic testing, contact lenses, etc.). Recently, the DTC option has become available in orthodontics with companies advertising at-home aligners. Their goal is to assist patients in receiving orthodontic treatment from home at a reduced cost by eliminating in-office visits involving face-to-face oversight of a dental professional. Because this treatment modality has appeared so recently, limited research exists assessing the overall satisfaction of patients undergoing DTC orthodontic treatment. With the options of traditional braces (B), in-office aligners (IOA), and DTC aligners (DTCA), many individuals are seeking answers on YouTube as to which method they might prefer. YouTube provides an exciting medium to examine the available orthodontic content and sentiment of potential, former, and current orthodontic patients.

The aim of this study was to 1) investigate and compare the educational value of the most popular YouTube orthodontic patient testimonials between braces, in-office aligners, and DTC aligners, and to 2) classify the emotional response of the viewers through a sentiment analysis of the video comments. 


\section{Methods}

The Virginia Commonwealth University Institutional Review Board deemed this study not applicable for review since it did not meet the criteria for human subjects research, since all information about the individuals was already publicly available. YouTube was searched on two separate occasions, two weeks apart on March 31 $1^{\text {st }}, 2019$, and April $14^{\text {th }}, 2019$. Before searching, the browser history and cookies were deleted to prevent a biased search due to Google's placement and personalized advertisement targeting. The browser was also placed in "private" mode.

A preliminary YouTube search provided useful information to determine how to obtain the most relevant results for each of the three orthodontic treatment modalities: braces (B), inoffice aligners (IOA), and direct-to-consumer aligners (DTCA). The most searched orthodontic companies providing in-office aligners and direct-to-consumer aligners were Invisalign ${ }^{\circledR}$ and Smile Direct Club ${ }^{\mathrm{TM}}$, respectively. As such, the three different phrases searched were "braces experience," "Invisalign experience," and "Smile Direct Club experience." The "sort by relevance" search filter was used, which is the YouTube search filter default and the most commonly used filter by viewers. ${ }^{25}$

Many studies have shown that approximately $90 \%$ of YouTube users click on videos displayed within the first three pages of search result, or the first $20-30$ videos. ${ }^{26}$ Therefore, the YouTube video ID of the first 50 videos for each of the three searches, for each day, were saved. The video searches between the two different days were screened for any duplicates. The videos were assessed by two examiners. The independent rater scored a subset of 12 videos on the subjective metrics to determine the interrater reliability of the primary investigator's classification of the videos. The following exclusion criteria for the videos were applied: video in 
non-English language, testimonials from non-patients, duplicates, videos without comments, poor audio-visual quality, no personal testimonial, and irrelevant information. Once this initial video screening was completed and after they were organized on increasing popularity order, the top 20 videos that met the proposed criteria were selected for each of the three orthodontic searches, totaling 60 videos for the study.

We leveraged the YouTube data Application Programming Interface (API) to extract the following 13 video metrics from each of the 60 videos: 1) Video ID, 2) title, 3) time since upload, 4) view count, 5) number of likes, 6) number of dislikes, 7) vlogger name, 8) vlogger subscription count, 9) vlogger view count, 10) number of comments, 11) duration of video, 12) claim of sponsorship, and 13) inclusion of before and after photos.

In addition, each video received an information completeness score (ICS) in order to assign an educational or qualitative value to each of them. ${ }^{7,27,28}$ This score is based off of the inclusion or lack of specific criteria that have been shown to be important and informative for healthcare consumers as they make personalized health decisions based off of social media information. Both examiners assigned a score from 1 to 5 for each video. For each topic mentioned by the vlogger, one point was earned to make up the ICS: 1) Treatment provider (orthodontist, general dentist, etc.), 2) cost of treatment, 3) treatment procedure details, 4) complications of treatment, and 5) the comparison to other treatment modalities.

Finally, the viewers' comments for each of the 60 videos were filtered and extracted from YouTube using the site's API. In order to avoid misinterpretation of the video comments for the analysis, only comments that included the word "braces," "Invisalign," "Smile Direct Club," or "SDC" were analyzed from each of their respective categories. After the comments were retrieved and advertisements were filtered out, they were uploaded into SentiStrength, an opinion 
mining software tool that has been utilized in previous studies, which generates a report of positive and negative sentiment scores. ${ }^{29-31}$ As a lexicon-based classifier, SentiStrength uses non-lexical linguistic rules and information to detect sentiment strength in short informal English text. Specifically, it measures the strength of positive and negative sentiment in English text and scores each on a scale of \pm 1 (neutral) to \pm 5 (extremely positive/extremely negative). For example, a text scored with +5 and -3 would indicate strongly positive and moderately negative emotions and would have an overall positive sentiment score. SentiStrength has been shown to perform well across social web data sets and is frequently used for YouTube video comment analyses. ${ }^{32}$

The comments were then categorized into positive, negative, or neutral classes. To do so, positive and negative scores were summed together. Comments whose scores summed to greater than 0 were denoted as a positive class, while those that summed to less than 0 were denoted as a negative class. In the case where scores summed to 0 , comments were denoted as neutral. That is, there was not a clear indication from the sentiment analysis whether the comment provided by the viewer had predominantly positive or negative sentiment.

Lastly, word clouds were generated for each treatment modality by positive and negative comments for the top 200 most frequent words using the wordcloud package (version 2.6). ${ }^{33}$ Rudimentary text analysis was performed to sort the comments. Punctuations, numbers, words of length 1, and stop words (“a”, "an”, "the”, etc.) were all removed. Additionally, specific words including "get", "getting", "got", "just", "can", "now", "im", "will", "braces", "invisalign", "direct", "club", "sdc", and "teeth" were removed prior to creating the word cloud. 


\section{$\underline{\text { Statistical Methods }}$}

Inter-rater agreement for subjective scoring was assessed with Cohen's Kappa.

Descriptive statistics were used to describe key findings within the data. Number of comments, likes, and dislikes were all standardized to the total view count by calculating the comments, likes, or dislikes per 1,000 views. The viewer interaction score was calculated based on Lena and Dindaroglu as the number of likes minus the number of dislikes divided by the total views. ${ }^{17}$ Association between video metrics and the overall ICS score were compared using Spearman Correlations initially with the goal of constructing an overall model for ICS score with any factors that were significantly associated at bivariate level. Due to non-normality of the data, metrics were compared across the treatment type using Kruskal Wallis test with Dwass, Steel, Critchlow-Fligner multiple comparison procedure for post hoc pairwise comparisons. For the sentiment analysis, a Chi-square test of independence was performed to assess the association between treatment modality and class of comment. The neutral class of comments were removed from statistical analysis.

A significance level was set at 0.05. SAS EG v.6.1 was used for all analyses. 
Figure 1. Video selection process for braces (B)

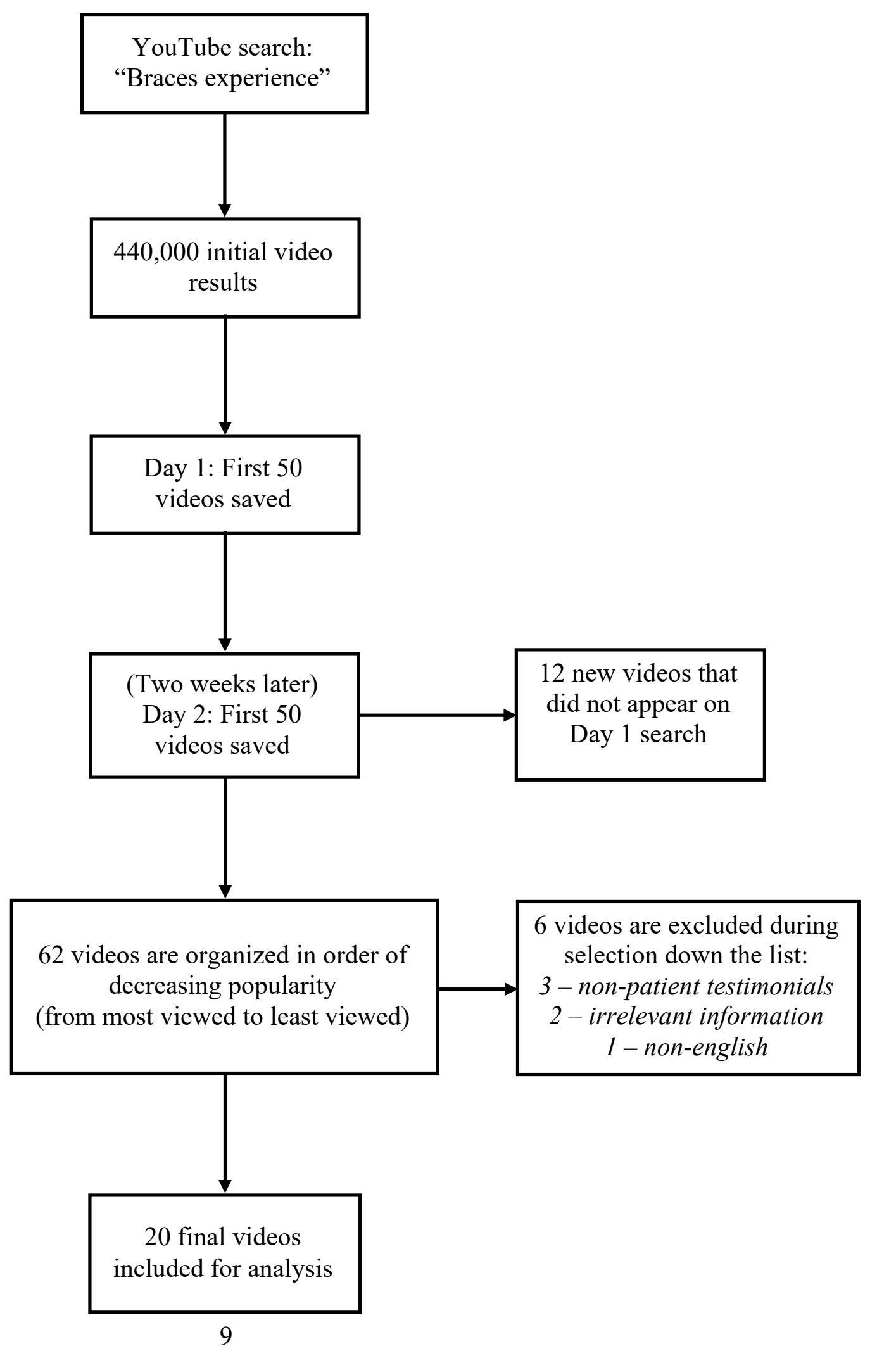


Figure 2. Video selection process for in-office aligners (IOA)

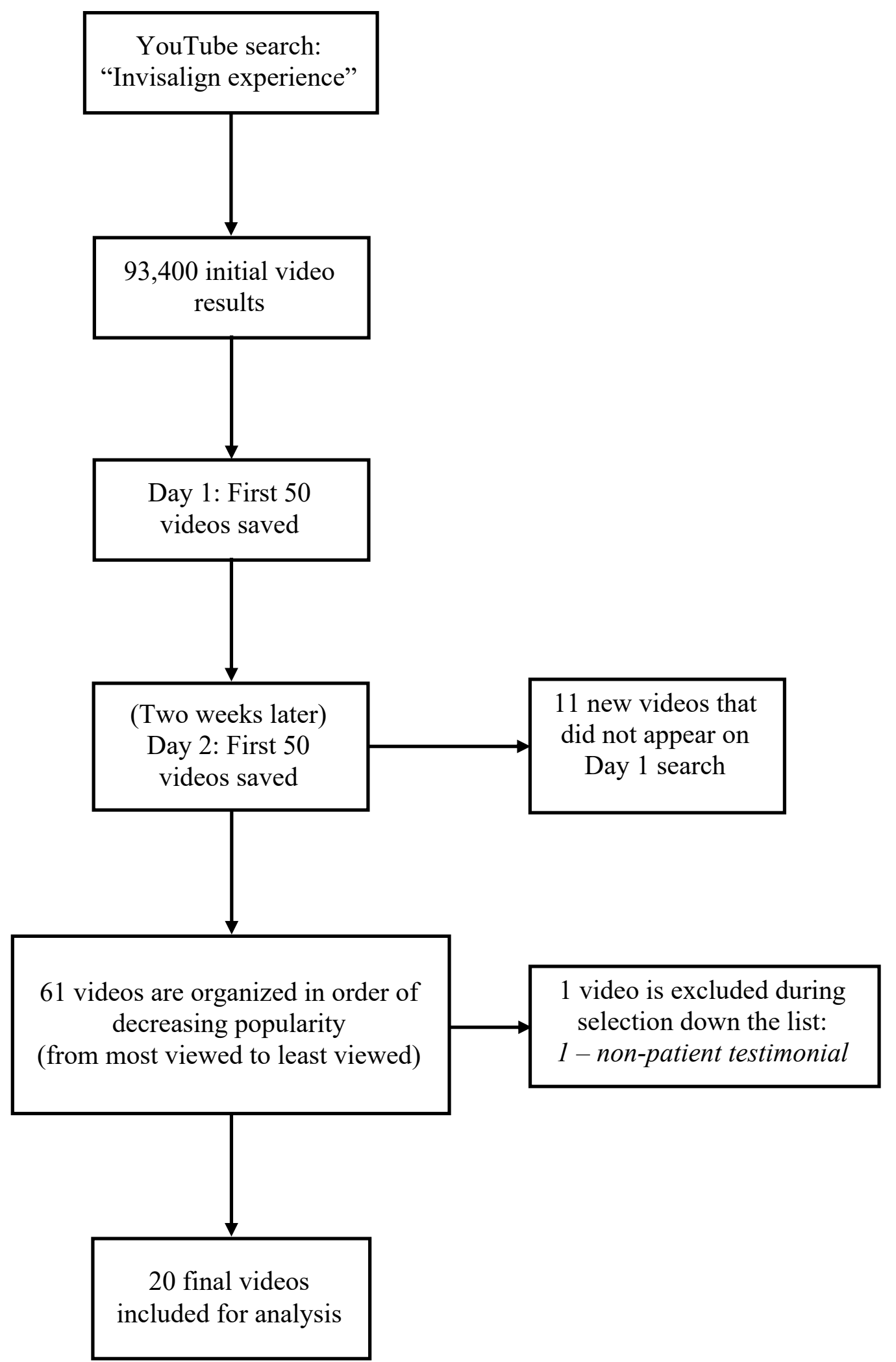


Figure 3. Video selection process for direct-to-consumer aligners (DTCA)

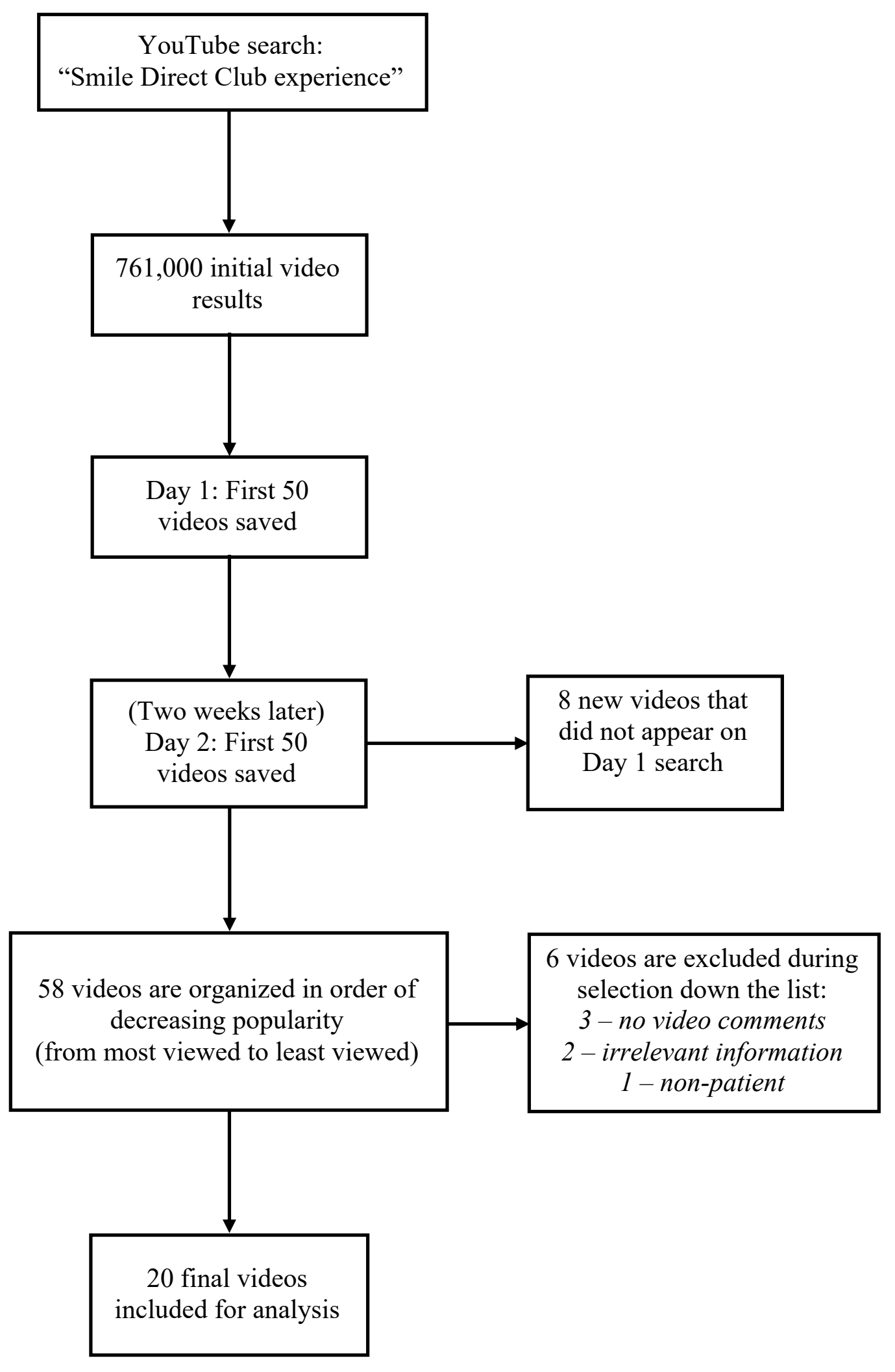




\section{Results}

The initial YouTube search on March 31 st 2019 , yielded a total of 1,294,400 video results: 440,000 for "braces experience", 93,400 for "Invisalign experience", and 761,000 for "Smile Direct Club experience." The YouTube search engine reported a similar total estimate for the search on April 14 $4^{\text {th }}, 2019$.

On both search days, the first 50 videos for each of the three searches were selected. The same procedure was repeated two weeks later, demonstrating slight differences between the first 50 videos that appeared from the searches from the two days. Figures 1-3 are flow charts of the final selection process.

Table 1. Summary of statistics on video metrics

\begin{tabular}{|lrr|}
\hline Variable & Median & IQR \\
\hline Information Completeness Score (out of 5) & 4 & $(3,4)$ \\
Video Duration (in minutes) & 592.50 & $(411.5,774)$ \\
Time Since Posted (days) & 611.50 & $(378,1229)$ \\
View count & 214,398 & $(88190,529700)$ \\
Number of comments & 346 & $(176,1014.5)$ \\
Number of dislikes & 133 & $(48.5,292.5)$ \\
Number of likes & 2,032 & $(640.5,7798)$ \\
Views per Day Posted & 310.99 & $(117,899.49)$ \\
Comments per 1,000 Views & 1.87 & $(1.2,3.02)$ \\
Likes per 1,000 Views & 9.98 & $(5.98,18.8)$ \\
Dislikes per 1,000 Views & 0.53 & $(0.41,0.8)$ \\
Viewer Interaction Score & 0.94 & $(0.51,1.81)$ \\
\hline
\end{tabular}

A total of 60 videos were analyzed and given an Information Completeness Score (ICS). Subjective video scoring was validated against an independent rater and agreement was determined to be 0.89 (95\% CI: 0.81-0.96). Based on these results, the primary investigator 
reviewed and scored the remaining videos. The median ICS for each video was 4 out of 5 . The lowest score given for any video was a 2 , with the highest being a 5 .

The median length for each video was 9 minutes and 53 seconds. The shortest video duration was 2 minutes and 22 seconds, while the longest was 24 minutes and 6 seconds. The median view count for each video was 214,398, with a median of 346 comments, 2,032 likes, 133 dislikes. In order to account for the time since the video was uploaded, the views were standardized to the number of days since the video had been posted resulting in a median views per day of 311. Summary values are given in Table 1.

The median subscriber count for all of the vloggers included in our study was 26,246. There was also a positive correlation between the number of subscribers and the number of views a vlogger's testimonial video received $(0.571, \mathrm{p}$-value $<0.0001)$. Interestingly, the directto-consumer aligner (DTCA) videos were posted by vloggers with significantly less subscribers than the other two groups (p-value $<0.001)$.

Six of the videos (10\%) made a claim of sponsorship. Four of those claims were from inoffice aligner (IOA) videos, along with one braces (B) video and one direct-to-consumer aligner (DTCA) video. However, this difference was not statistically significant ( $p$-value $=0.3438$ ). The 60 videos were created by 54 unique vloggers.

Analyzing the criteria of the ICS more in depth, zero of the 20 DTCA vloggers mentioned their treatment provider (dentist or orthodontist), while 18 of the 20 IOA vloggers and 16 of the 20 braces vloggers mentioned their treatment provider. For those that did mention their treatment provider, 27 of them reported being treated by an orthodontist (14 braces and 13 Invisalign), while 7 of them reported being treated by a general dentist ( 2 braces and 5 
Invisalign). The IOA vloggers also more frequently made comparisons to other treatment modalities $(\mathrm{B}=5 / 20, \mathrm{IOA}=18 / 20, \mathrm{DTCA}=16 / 20)$.

DTCA vloggers mentioned their treatment cost more frequently than the other two groups $(B=6 / 20, I O A=11 / 20, D T C A=14 / 20)$. All three groups mentioned treatment procedure details and any presence or lack of complications of treatment equally, with every vlogger among all three treatment modalities mentioning the pain aspect of orthodontic treatment.

A majority of the videos (38 of the 60) included before/after photos of the vlogger's orthodontic treatment. In the B and IOA video groups 12 out of the 20 videos included before/after photos, while in the DTCA video group 14 out of the 20 included before/after photos. This difference between treatment modalities was not statistically significant.

Table 2. Association between video metrics and ICS

\begin{tabular}{|lrr|}
\hline Association with Total Score & $\begin{array}{r}\text { Spearman } \\
\text { Correlation }\end{array}$ & $\begin{array}{r}\text { p-value* } \\
\hline \text { Video Duration (in minutes) }\end{array}$ \\
Time Since Posted (days) & 0.245 & 0.0592 \\
View count & -0.029 & 0.8250 \\
Number of comments & 0.091 & 0.4869 \\
Number of dislikes & 0.004 & 0.9745 \\
Number of likes & 0.089 & 0.4983 \\
Views per Day Posted & 0.005 & 0.9724 \\
Comments per 1000 Views & 0.106 & 0.4184 \\
Likes per 1000 Views & -0.040 & 0.7603 \\
Dislikes per 1000 Views & -0.089 & 0.4988 \\
Viewer Interaction Score & -0.048 & 0.7151 \\
\hline
\end{tabular}

$*$ P-value for test for Spearman Correlation $\neq 0$

In comparing associations between the video metrics and the Information Completeness Score (ICS), there was a marginally significant association between the total video duration and the ICS $(r=0.25, p$-value $=0.0592)$, indicating that as the length of a video increased, it became 
more likely to have a higher Information Completeness Score. None of the other video metrics were significantly correlated with the ICS (p-value $>0.40)$; Spearman correlations were all less than 0.1. Associations between video metrics and ICS are given in Table 2.

There was, however, a significant association between the Information Completeness Score (ICS) and the treatment type (p-value=0.0005; Table 3). Specifically, in-office aligner (IOA) videos had a higher median ICS than the braces (B) or direct-to-consumer aligner (DTCA) videos. IOA videos had a median score of 5 , with 11 of the 20 videos achieving the highest score on the ICS. DTCA videos were most likely to score a 4 out of $5(n=12,60 \%)$ and B videos most often scored a 3 out of $5(n=11,55 \%)$.

Treatment type was also associated with significant differences in the total view count (pvalue $=0.0002)$, number of comments ( $p$-value $<0.0001)$, number of likes ( $p$-value $<0.0001)$, and number of dislikes ( $p$-value $=0.0009$ ). After adjusting for the number of days since the video was posted, there was still a significant difference in views per day posted across the three treatment types ( $p$-value $=0.0255)$. Braces videos had the most views per day posted among the three groups. This difference was significant when comparing against DTCA videos (adjusted pvalue $=0.0343$ ) but was not significant when comparing against IOA videos (adjusted pvalue $=0.5763)$.

For every 1,000 views that each video garnered, the number of likes was significantly higher for B videos than DTCA (adjusted p-value=0.0048) and IOA (adjusted p-value=0.0488) videos; and the viewer interaction score was also significantly higher for B videos than for the other two groups. Summary of the differences by treatment type are provided in Table 3. 
Table 3. Associations between video metrics across treatment type

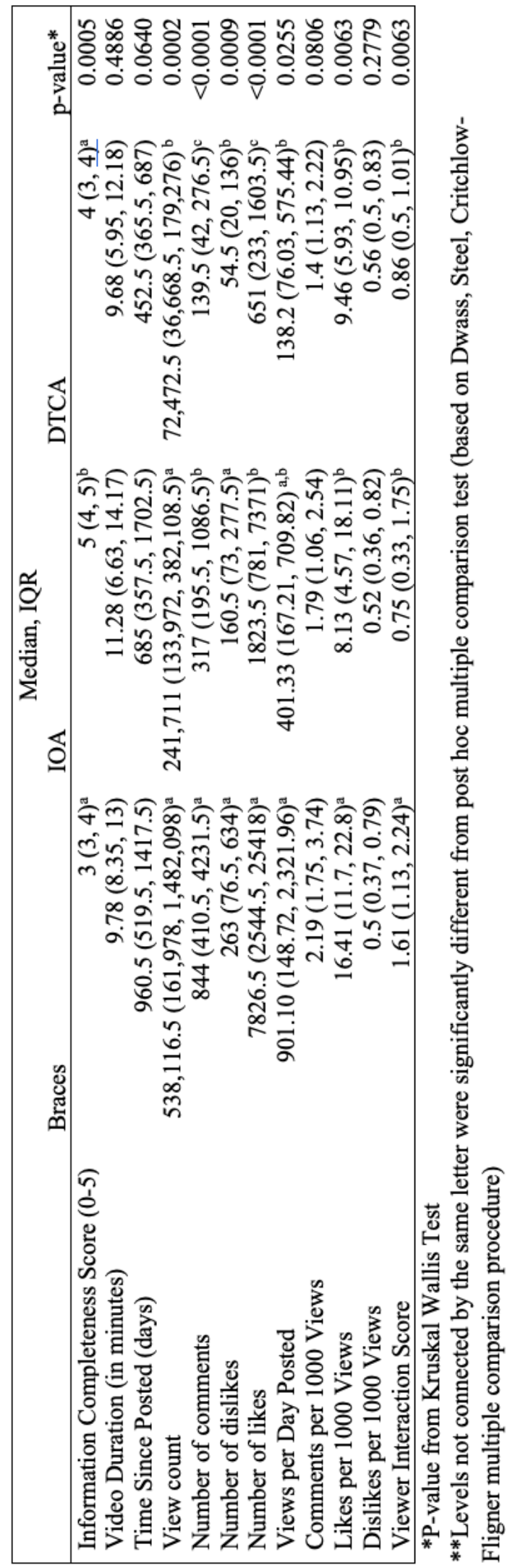




\section{$\underline{\text { Sentiment }}$}

There were 66,116 comments posted by viewers for all 60 videos. The majority of the comments (70\%) came from the videos about treatment with braces (B).

A total of 8,438 qualifying comments were collected from the 60 videos analyzed in this study. The majority of comments were in response to the B videos with 5,766 (65.3\%), while IOA and DTCA videos had 2,418 (28.7\%) and 254 (3.0\%) comments each. After categorizing the comments into classes, 3,289 comments were identified as neutral and were removed from statistical analyses.

For the remaining comments, there were significantly more positive comments than negative (p-value $<0.0001$ ). Of the 5,149 comments with polarity, there were 2,762 positive $(53.6 \%)$ and 2,387 negative (46.4\%) comments. The positive comments resulted in an average score of 1.49 (scored from +1 to +5$)(\mathrm{SD}=0.64)$ and the negative comments resulted in an average score of -1.75 (scored from -1 to -5$)(\mathrm{SD}=0.79)$, respectively. These data indicate that while there were more positive comments than negative comments for all 60 videos, the negative comments were on average stronger in sentiment than the positive comments.

When comparing comment sentiment between the three different treatment modalities, results from the Chi-square test of independence indicated that there was not a significant association between the treatment modality and positive or negative comments $(p=0.5679)$. A summary of the relationship between treatment modality and comment sentiment is provided in Table 4.

There were 1,838 (53.1\%) B video comments that were categorized as positive. The most frequent terms included "like", "look", "video", "really," and "love" (See Figure 4). There 
were 833 (54.8\%) IOA and 91 (53.8\%) DTCA video comments that were categorized as positive. The most frequent terms were found to be similar to that of the positive braces video comments.

In contrast, there were 1,621 (46.9\%) B video comments that were categorized as negative. The most frequent terms included words like "hurt", "bad", "pain", and "hate". There were 688 (45.2\%) IOA and 78 (46.2\%) DTCA video comments that were categorized as negative. For negative IOA videos, the most frequent terms were found to be similar to that of the negative B video comments. However, for DTCA video comments categorized as negative, the most frequent terms included words like "bite", "treatment", "dentist", "orthodontist", and "results" (See Figures 5-8).

Table 4. Relationship between treatment modality and comment sentiment

\begin{tabular}{|l|r|r|r|}
\hline & \multicolumn{2}{|c|}{ Comment Class } & \\
\cline { 1 - 3 } Treatment Modality & $\begin{array}{r}\text { Positive } \\
(\mathbf{N}=\mathbf{2 7 6 2})\end{array}$ & $\begin{array}{r}\text { Negative } \\
(\mathbf{N}=\mathbf{2 3 8 7})\end{array}$ & \multirow{2}{*}{ p-value } \\
\hline Braces (B) & $1838(53 \%)$ & $1621(47 \%)$ & 0.5679 \\
\cline { 1 - 2 } In-office aligners (IOA) & $833(55 \%)$ & $688(45 \%)$ & \\
\cline { 1 - 2 } Direct-to-consumer aligners (DTCA) & $91(54 \%)$ & $78(46 \%)$ & \\
\hline
\end{tabular}




\section{Discussion}

As the most popular video-sharing website in the world, YouTube serves to inform, entertain, and publicize individuals, businesses, artists, commentators, and even healthcare researchers. ${ }^{2}$ Publicly available insight from social media users can be gathered from YouTube and analyzed on a very large scale. Traditional survey-based approaches are being partially superseded by social media data mining to help reduce the experimenter and memory bias inherent in surveys. ${ }^{32}$ A social media platform like YouTube facilitates researchers in gathering and interpreting spontaneous data collected from individuals who post, watch, and comment on YouTube videos. While investigating the effectiveness of antismoking campaign videos, Chung et al. detailed the incredible power of social media research in the healthcare industry by analyzing the video metrics and the viewers' reactions to the YouTube videos, which were predominantly positive. ${ }^{34}$ Noll et al. showed that similar techniques can be applied to the orthodontic field, demonstrating that social media users expressed more positive than negative sentiment about orthodontic treatment. ${ }^{10}$

This study expounded on the findings of Livas et al. who concluded that YouTube viewers' comments regarding orthodontic treatment with Invisalign ${ }^{\circledR}$ were significantly more

positive than negative. ${ }^{27}$ In the current study, we compared three different treatment modalities, executed a specific content breakdown and video metrics comparison, and performed a detailed text analysis supplemented with word clouds to help visualize the data.

The 60 videos included in this study were viewed 34,384,786 times by internet users throughout the world. This figure, along with the total number of likes, dislikes, and video comments, confirms an extremely high rate of activity among orthodontic patient testimonial videos on YouTube. The median video duration was surprisingly long at 9 minutes and 53 
seconds; previous consumer research has shown that videos under 5 minutes in duration will generally garner the most user engagement. ${ }^{35}$ However, our results show that the correlation between the viewer interaction score ((\# of likes - \# of dislikes)/total views)) and the duration of the video was not significant. Viewers were just as likely to interact with longer videos as they were with shorter, more concise videos.

While systematically investigating online healthcare information, Madathil et al. detailed that YouTube is increasingly being used as a platform for disseminating health information, but often contains misleading information that is primarily anecdotal. ${ }^{36}$ The Information Completeness Score (ICS) allowed us to measure the informative value, rather than the entertainment value, of the patient testimonials by assessing whether the videos mentioned the 1) treatment provider, 2) cost of treatment, 3) treatment procedure details, 4) complications of treatment, and 5) any comparison to other treatment modalities. Surprisingly, there was no correlation between a higher ICS score and any of the main video metrics (duration, view count, number of comments, etc.). These results might indicate that the information vs. entertainment value of a video does not seem to play a significant role in the viewing behavior of the YouTube audience for videos on orthodontic treatment.

Comparing, however, the Information Completeness Score among the three different treatment modalities revealed a statistically significant difference, as the in-office aligner (IOA) videos scored consistently higher than the braces and DTCA videos. These results indicate that the Invisalign ${ }^{\circledR}$ vloggers were more likely to include information about their orthodontic experience that might prove to be helpful in the potential patient's decision-making process. Specifically, the IOA vloggers mentioned more frequently their treatment provider as well as comparisons to braces and direct-to-consumer aligner treatment. 
One of the supposed benefits of orthodontic treatment with direct-to-consumer aligners is the elimination of in-office visits, which can potentially lead to time-saving and convenience, but can also lead to a diminished relationship with the treatment provider and potential harm due to the lack of professional supervision. Even though every Smile Direct Club $^{\mathrm{TM}}$ patient receives the name of their treating doctor, who is licensed in the state in which the patient resides, the lack of face-to-face interaction with their provider could explain why none of the DTCA vloggers mentioned the dentist overseeing their treatment, despite having their provider's name printed on the aligner bags. ${ }^{37}$ This procedural difference might play a role in the final orthodontic (patient) outcome. A recent survey of adult patients who had completed active treatment with in-office aligners found that the doctor-patient relationship was highly associated with the level of overall treatment satisfaction reported. ${ }^{38}$

For the 7 vloggers who claimed to be treated by general dentists, 2 were treated with braces and 5 were treated with Invisalign ${ }^{\circledR}$, which is consistent with industry trends. ${ }^{39}$ Prior to the inception of Invisalign ${ }^{\circledR}$ in 1998, the principal orthodontic treatment modality was fixed appliances with braces. Clear aligners have continued to increase in popularity, and at the start of 2018, accounted for $15 \%$ of the existing orthodontic appliances market in the United States. ${ }^{40}$ An increase in demand for orthodontic treatment with clear aligners has coincided with an increase in general dentists providing orthodontic treatment. Studies report that $18-20 \%$ of general dentists provide routine comprehensive orthodontic treatment and $32-57 \%$ offer some form of limited orthodontic treatment. ${ }^{41,42}$

Previous studies on the impact of before and after photos for healthcare treatment have shown that the attitude, risk perception, and purchase intention toward a procedure or product are highly influenced by the visual contrast and complexity provided by the photos. ${ }^{43}$ The inclusion 
of these photos seems to be important for the YouTube audience seeking orthodontic treatment as they were included in $63 \%$ of the videos in our study, with no difference between groups. The vloggers for all three treatment modalities were equally as likely to include photos documenting their orthodontic journey.

Despite a surge in popularity with clear aligner treatment, largely due to the advent of direct-to-consumer orthodontics, braces have continued to remain the principal orthodontic treatment modality. Our results indicate that the YouTube audience is engaging more with braces patient testimonial videos than with clear aligner patient testimonial videos. When comparing among the three treatment modalities, the braces videos had significantly more likes, comments, and a higher viewer interaction score than the IOA and DTCA videos.

Our sentiment analysis revealed no difference in positive and negative sentiment between the video comments for the three different treatment modalities (B, IOA, DTCA). This finding is consistent with Noll et al. who found no significant difference in sentiment between tweets about braces and tweets about Invisalign ${ }^{\circledR} .{ }^{10}$ Although the unsolicited comments from the 60 videos in our study have the potential to be targeted at topics irrelevant to the orthodontic patient experience, we reduced the potential for comment misinterpretation by only selecting comments that contained the words "braces," "Invisalign," "Smile Direct Club, or "SDC." This allowed us to analyze the sentiment of individual YouTube viewers who might be interested in, currently undergoing treatment in, or finished with treatment in one of these three orthodontic treatment modalities.

Align technology has advertised Invisalign ${ }^{\circledR}$ treatment as offering an improved patient experience over braces, as well as over other brands of clear aligners. ${ }^{44}$ Miller et al. found that Invisalign ${ }^{\circledR}$ patients experienced less discomfort when compared to braces, while Shalish et al. 
found more mixed experiences between braces and Invisalign ${ }^{\circledR}$ patients. ${ }^{45,46}$ The results of this YouTube study did not support any difference in the sentiment from the viewers' comments between the three treatment modalities.

Smile Direct Club $^{\mathrm{TM}}$ (SDC), founded in 2014, is a dental support organization that claims to have pioneered a teledentistry platform for orthodontic treatment. ${ }^{37}$ As of October 2019 , more than 750,000 patients have started treatment through their direct-to-consumer platform. They report an $87 \%$ positive sentiment rating among different consumer sites (BBB, Yelp, Google, etc). The comments analyzed in our study give insight into the joys and frustrations of orthodontic treatment. Interestingly, when comparing the most frequent terms found in comments that were classified as negative, there was a stark difference between the direct-toconsumer aligner (DTCA) group and the other two groups. For the braces and in-office aligner (IOA) videos, the words most commonly included in the negative comments were consistent with other dental sentiment analysis studies: "pain," "hurt," "bad," and "scared." 10 For the direct-to-consumer aligner (DTCA) videos, words like "bite," "treatment," "dentist," and "results" were most commonly included. In addition, the words "money" and "refund" were only recurrently found in the DTCA group and were each present in over $15 \%$ of the negative comments (See Figures 5-8). This could be explained by the fact that DTC orthodontic companies focus their marketing on cost-savings and the convenience of their product delivery, instead of the overall orthodontic experience. Orthodontic providers need to have a thorough understanding of these common negative reactions to treatment in order to improve the patient experience.

Overall, the majority of viewers' comments expressed positive sentiment. The most commonly used words in the comments classified as positive were "like," "look," "video," and 
"love." Many viewers seemed to comment on their own orthodontic journey or the journey of the vlogger by using words like "excited," "want," "going," "need," and "soon." A unique aspect of orthodontics is the amount of time required to complete treatment. Words like "years," "months," and "time" were frequently used to express the commitment necessary to achieve the desired results. Finally, the words "thank" and "thanks" were repeatedly used to potentially express appreciation for their new smile or appreciation to the vlogger for the informative patient testimonial. This finding demonstrates that finishing orthodontic treatment is an important day in the life of patients. Additionally, these moments and these videos are important for the orthodontic practice, as social media can be viewed as the preeminent source for word-of-mouth marketing strategies.

A marketing strategy gaining traction in numerous industries is called influencer marketing, which goes hand-in-hand with social media, content, and word-of-mouth marketing. This tactic focuses on using key leaders, or individuals with a strong social media presence, to drive a brand's message to the larger market. By posting engaging content and interacting with their audience on YouTube, successful vloggers can attract followers to regularly watch and subscribe to their videos, allowing the followers to be notified every time the vlogger posts additional content. Our results indicate a positive correlation between subscriber count and view count, implying that a video posted by a vlogger with more subscribers is more likely to end up with a higher total view count. We examined a potential sponsorship of the YouTube vloggers by orthodontic companies or businesses to post these videos in an attempt to influence potential orthodontic patients. Along with carefully analyzing the video content, we searched the description field of each video looking for sponsorship statements or endorsement deals. We 
only found that 6 of the 60 videos (10\%) made public claims of sponsorship. For those that were sponsored, the orthodontic company itself or the treatment provider provided the incentive.

This study investigated and compared metrics, trends, and sentiment among YouTube users interacting with three common orthodontic treatment modalities (braces, IOA, and DTCA). Properly executed research in healthcare can help provide a valuable glimpse inside the minds of patients. ${ }^{47}$ Analyzing naturally occurring data helped strengthen the validity of our study. In addition, we used SentiStrength as our sentiment analysis tool, which has been shown to perform significantly above the baseline for correlation across social web data sets. ${ }^{48}$

With over 400 hours of video uploaded to YouTube every minute, a limitation inherent to our study involves the dynamics of YouTube and its ever-changing data. New orthodontic patient testimonial videos will continue to surface providing new data that could be analyzed. Additionally, an assumption was made that any video comment analyzed in our study was about the orthodontic patient experience. Some comments could have been posted by YouTube users with no interest in orthodontics merely expressing their thoughts on the different orthodontic treatment modalities.

This study demonstrated a way to utilize and compare the abundance of publicly available information on social media platforms like YouTube. It can help provide insight to orthodontic providers as they seek to understand the current orthodontic landscape and meet the needs of their patients. It can also serve as valuable information to the leaders of professional organizations as they advocate for the interests of their members. Future studies can utilize similar methods to examine other aspects of the orthodontic patient experience. 


\section{Conclusion}

1. There is high user engagement on YouTube with orthodontic patient testimonials

2. Braces videos have significantly more likes, comments, and a higher viewer interaction score than the in-office aligner and direct-to-consumer aligner videos

3. In-office aligner videos had a higher median Information Completeness Score than the braces or direct-to-consumer aligner videos

4. YouTube viewers' comments on orthodontic patient testimonials express more positive sentiment than negative sentiment

5. There is no significant difference in positive and negative sentiment between the video comments for the three different treatment modalities

6. Positive orthodontic-related video comments often highlight gratitude for a great smile and excitement to begin or end the orthodontic treatment journey 


\section{References}

1. Fagerlin A, Wang C, Ubel PA. Reducing the influence of anecdotal reasoning on people's health care decisions: Is a picture worth a thousand statistics? Med Decis Mak. 2005;25(4):398-405. doi:10.1177/0272989X05278931

2. YouTube.com. YouTube for Press. https://www.youtube.com/about/press/. Published 2019. Accessed December 10, 2019.

3. CISCO 2017. "The Zettabyte Era: Trends and Analysis", whte paper. Cisco 2017. 2017;(June):1-29. doi:1465272001812119

4. Hawke B, Przybylo AR, Paciulli D, Caulfield T, Zarzeczny A, Master Z. How to Peddle Hope: An Analysis of YouTube Patient Testimonials of Unproven Stem Cell Treatments. Stem Cell Reports. 2019;12(6):1186-1189. doi:10.1016/j.stemcr.2019.05.009

5. ReFaey K, Tripathi S, Yoon JW, et al. The reliability of YouTube videos in patients education for Glioblastoma Treatment. J Clin Neurosci. 2018;55:1-4. doi:10.1016/j.jocn.2018.07.001

6. Samuel N, Alotaibi NM, Lozano AM. YouTube as a Source of Information on Neurosurgery. World Neurosurg. 2017;105:394-398. doi:10.1016/j.wneu.2017.05.111

7. Nason K, Donnelly A, Duncan HF. YouTube as a patient-information source for root canal treatment. Int Endod J. 2016;49(12):1194-1200. doi:10.1111/iej.12575

8. Detels R, Gulliford M, Karim QA TC. New communication technologies, social media, and public health. In: Oxford Textbook of Global Public Health. 6th ed. Oxford: Oxford University Press; 2015:391.

9. Livas C, Delli K, Pandis N. "My Invisalign experience": content, metrics and comment sentiment analysis of the most popular patient testimonials on YouTube. Prog Orthod. 2018;19(1):1-8. doi:10.1186/s40510-017-0201-1

10. Noll D, Mahon B, Shroff B, Carrico C, Lindauer SJ. Twitter analysis of the orthodontic patient experience with braces vs Invisalign. Angle Orthod. 2017;87(3):377-383. doi:10.2319/062816-508.1

11. Kouloumpis E, Wilson T MJ. Twitter sentiment analysis: The good the bad and the omg! Icwsm. 2011;(11):538-541.

12. Morris G. Statitics from Invisalign NAKO. In: Head of Google Health Vertical. ; 2019.

13. Knösel M, Jung K. Informational value and bias of videos related to orthodontics screened on a video-sharing Web site. Angle Orthod. 2011. doi:10.2319/091710-541.1 
14. Guo J, Yan X, Li S, Van der Walt J, Guan G, Mei L. Quantitative and qualitative analyses of orthodontic-related videos on YouTube. Angle Orthod. 2020;00(00).

doi: $10.2319 / 082019-542.1$

15. Ustdal G, Guney AU. YouTube as a source of information about orthodontic clear aligners. Angle Orthod. 2020;00(00):1-6. doi:10.2319/072419-491.1

16. Hegarty E, Campbell C, Grammatopoulos E, DiBiase AT, Sherriff M, Cobourne MT. YouTube $^{\mathrm{TM}}$ as an information resource for orthognathic surgery. J Orthod. 2017;44(2):9096. doi:10.1080/14653125.2017.1319010

17. Lena Y, Dindaroglu F. Lingual orthodontic treatment: A YouTubee video analysis. Angle Orthod. 2018;88(2):208-214. doi:10.2319/090717-602.1

18. Birkeland K, Bøe OE, Wisth PJ. Relationship between occlusion and satisfaction with dental appearance in orthodontically treated and untreated groups. A longitudinal study. Eur J Orthod. 2000;22(5):509-518. doi:10.1093/ejo/22.5.509

19. M. Elizabeth Bennett, J.F. Camilla Tulloch, Katherin W. L. Vig CLP. Measuring Orthodontic Treatment Satisfaction: Questionnaire Development and Preliminary Validation. J Public Heal Dent. 2001;61(3):155-160.

20. Farishta S. Patient's Perceptions Regarding Orthodontic Needs and Satisfactory Level with the Procedure. J Int oral Heal JIOH. 2015;7(9):79-82.

21. Feldmanna I. Satisfaction with orthodontic treatment outcome. Angle Orthod. 2014;84(4):581-587. doi:10.2319/093013-710.1

22. Pacheco-Pereira C, Brandelli J, Flores-Mir C. Patient satisfaction and quality of life changes after Invisalign treatment. Am J Orthod Dentofac Orthop. 2018;153(6):834-841. doi:10.1016/j.ajodo.2017.10.023

23. Azaripour A, Weusmann J, Mahmoodi B, et al. Braces versus Invisalign $\AA$ : Gingival parameters and patients' satisfaction during treatment: A cross-sectional study. BMC Oral Health. 2015;15(1):1-5. doi:10.1186/s12903-015-0060-4

24. Ke Y, Zhu Y, Zhu M. A comparison of treatment effectiveness between clear aligner and fixed appliance therapies. BMC Oral Health. 2019;19(1):1-10. doi:10.1186/s12903-0180695-z

25. Sampson M, Cumber J, Li C, Pound CM, Fuller A, Harrison D. A systematic review of methods for studying consumer health YouTube videos, with implications for systematic reviews. PeerJ. 2013;1:e147. doi:10.7717/peerj.147

26. Knösel M, Jung K. Informational value and bias of videos related to orthodontics screened on a video-sharing Web site. Angle Orthod. 2011;81(3):532-539. doi:10.2319/091710541.1

27. Livas C, Delli K, Pandis N. "My Invisalign experience": content, metrics and comment sentiment analysis of the most popular patient testimonials on YouTube. Prog Orthod. 2018. doi:10.1186/s40510-017-0201-1

28. Bezner SK, Hodgman EI, Diesen DL, et al. Pediatric surgery on YouTube ${ }^{\mathrm{TM}}$ : Is the truth out there? J Pediatr Surg. 2014;49(4):586-589. doi:10.1016/j.jpedsurg.2013.08.004

29. Jain R. Sentiment Analysis on YouTube Movie Trailer comments to determine the impact 
on Box-Office Earning.

30. Krishna A, Zambreno J, Krishnan S. Polarity Trend Analysis of Public Sentiment on YouTube. Comad. 2013:125-128.

31. Asghar MZ, Ahmad S, Marwat A, Kundi FM. Sentiment Analysis on YouTube: A Brief Survey. 2015:1-11. doi:14.9831/1444-8939.2015/3-1/MAGNT.124

32. Thelwall M. Social media analytics for YouTube comments: potential and limitations. Int J Soc Res Methodol. 2018;21(3):303-316. doi:10.1080/13645579.2017.1381821

33. Fellows I. wordcloud: Word Clouds. 2018.

34. Chung JE. Antismoking campaign videos on YouTube and audience response:

Application of social media assessment metrics. Comput Human Behav.

2015;51(PA):114-121. doi:10.1016/j.chb.2015.04.061

35. Lella A. Comscore Releases U.S. Online Video Rankings. Comscore, Inc. https:/www.comscore.com/Insights/Press-Releases/2014/2/comScore-Releases-January2014-US-Online-Video-Rankings.

36. Madathil KC, Rivera-Rodriguez AJ, Greenstein JS, Gramopadhye AK. Healthcare information on YouTube: A systematic review. Health Informatics J. 2015;21(3):173-194. doi:10.1177/1460458213512220

37. Relations SM. Smile Direct Club issues statement on Organized Dentistry' s AntiCompetitive Legal Actions. 2019:4-6.

38. Pachêco-Pereira C, Pereira JR, Dick BD, Perez A, Flores-Mir C. Factors associated with patient and parent satisfaction after orthodontic treatment: A systematic review. Am J Orthod Dentofac Orthop. 2015;148(4):652-659. doi:10.1016/j.ajodo.2015.04.039

39. Galbreath RN, Hilgers KK, Silveira AM, Scheetz JP. Orthodontic treatment provided by general dentists who have achieved master's level in the Academy of General Dentistry. Am J Orthod Dentofac Orthop. 2006;129(5):678-686. doi:10.1016/j.ajodo.2004.10.018

40. TeleDentistry Association A. Teleorthodontics' Democratization of Clear Aligner Treatment. https://www.americanteledentistry.org/teleorthodontics-democratization-ofclear-aligner-treatment/. Published 2019.

41. Wolsky SL, McNamara JA. Orthodontic services provided by general dentists. Am J Orthod Dentofacial Orthop. 1996;110(2):211-217. doi:10.1016/S0889-5406(96)70111-7

42. Vicéns J, Russo A. Comparative use of invisalign $®$ by orthodontists and general practitioners. Angle Orthod. 2010;80(3):425-434. doi:10.2319/052309-292.1

43. Park SE. Uncover the magic: the use of before and after photos in weight loss product advertisements. Univ Alabama Libr. 2018.

44. Align Technology. The Advantage Over Braces. http://www.invisalign.com/whyinvisalign/advantage-over-braces. Accessed August 2, 2020.

45. Miller KB, McGorray SP, Womack R, et al. A comparison of treatment impacts between Invisalign aligner and fixed appliance therapy during the first week of treatment. $\mathrm{Am} \mathrm{J}$ Orthod Dentofac Orthop. 2007;131(3):302.e1-302.e9. doi:10.1016/j.ajodo.2006.05.031

46. Shalish M, Cooper-Kazaz R, Ivgi I, et al. Adult patients' adjustability to orthodontic 
appliances. Part I: A comparison between Labial, Lingual, and Invisalign ${ }^{\mathrm{TM}}$. Eur $J$ Orthod. 2012;34(6):724-730. doi:10.1093/ejo/cjr086

47. Bhamrah G, Ahmad S, Nimhurchadha S. Internet discussion forums, an information and support resource for orthognathic patients. Am J Orthod Dentofac Orthop.

2015;147(1):89-96. doi:10.1016/j.ajodo.2014.08.020

48. Saif H. Semantic Sentiment Analysis of Microblogs. 2015. 


\section{SUPPLEMENTARY FIGURES}

Figure 4. Most common words found in positive comments among all three groups
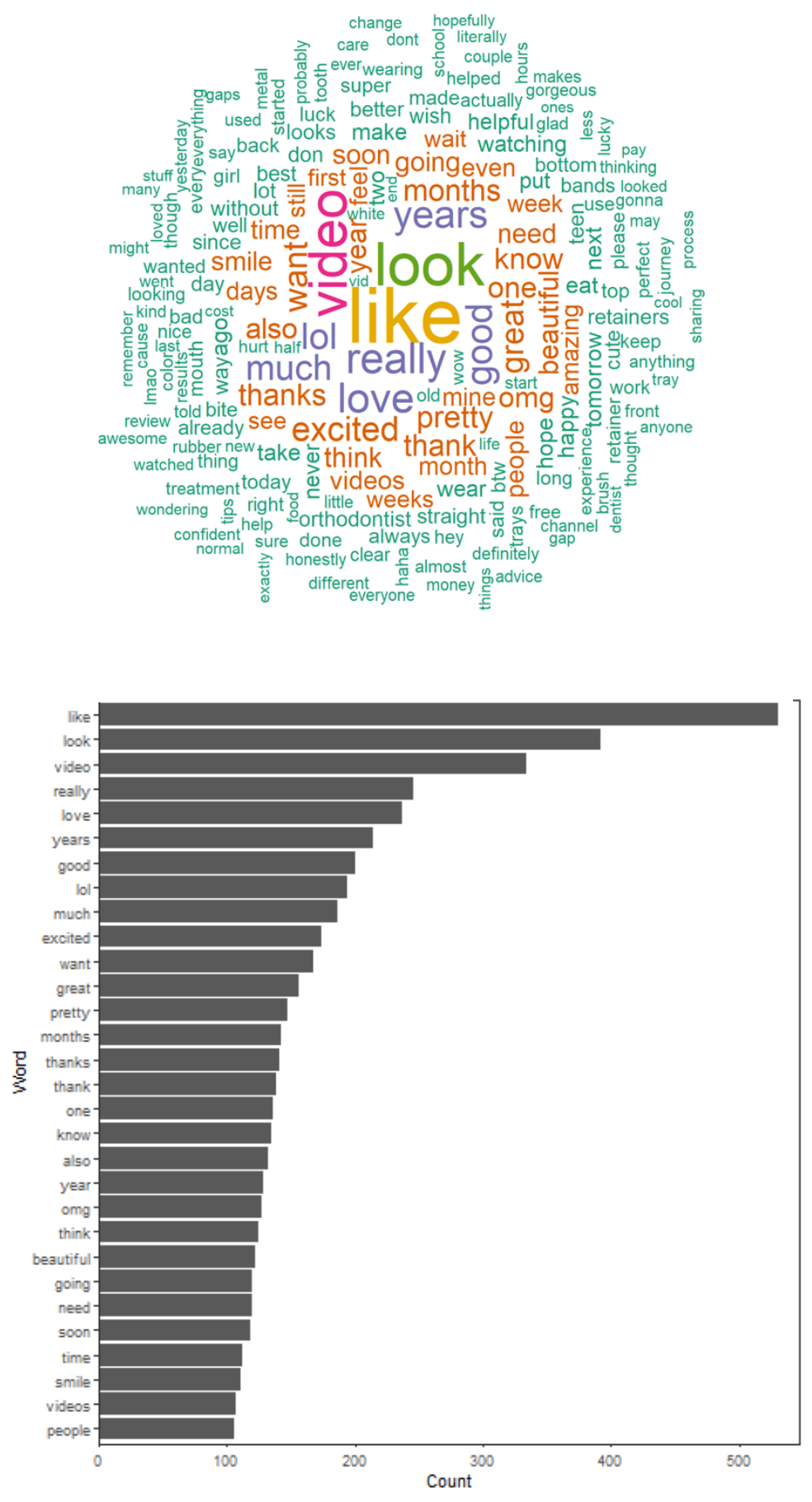
Figure 5. Most common words found in negative comments among all three groups
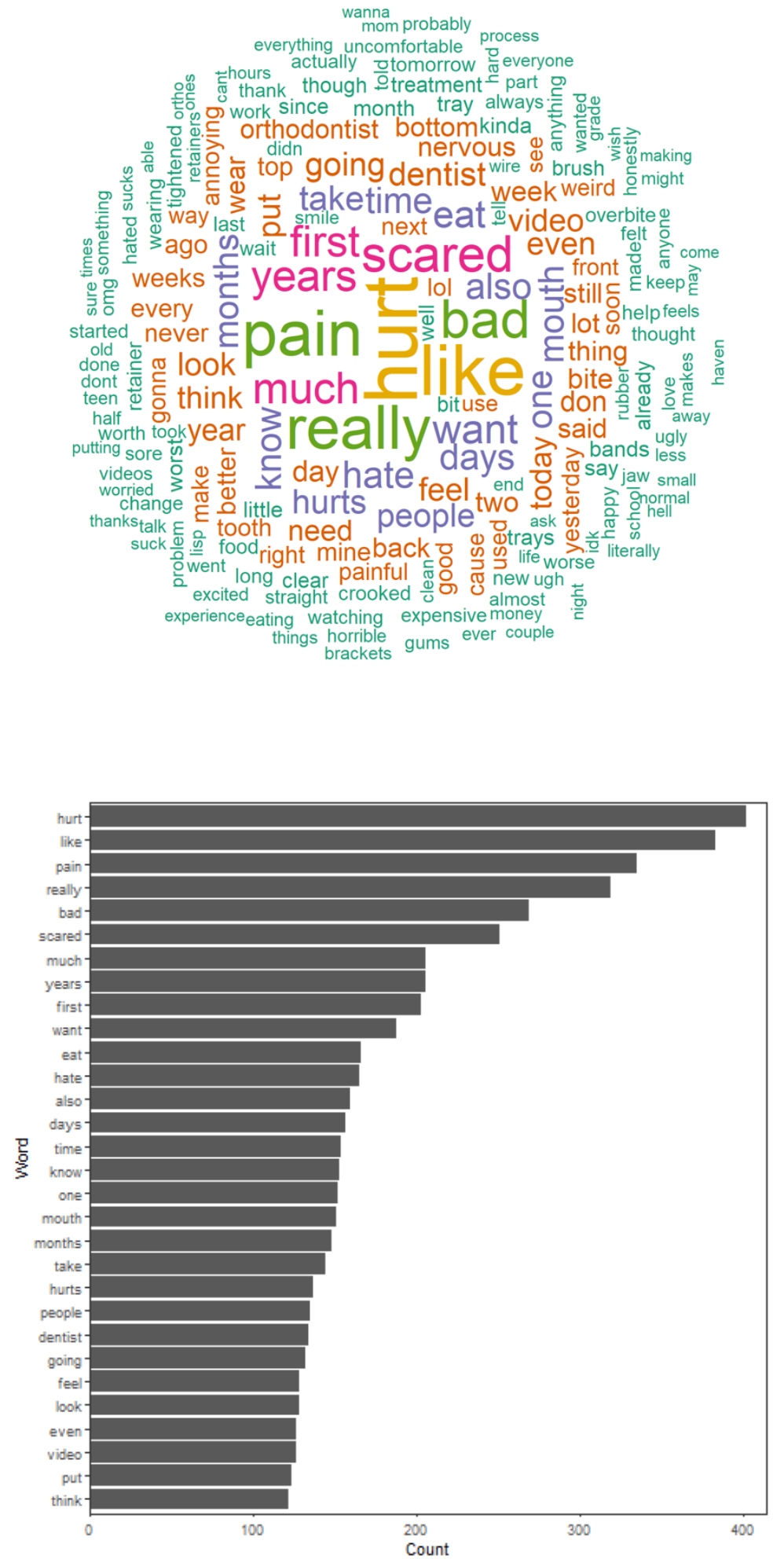
Figure 6. Most common words found in negative comments from B videos
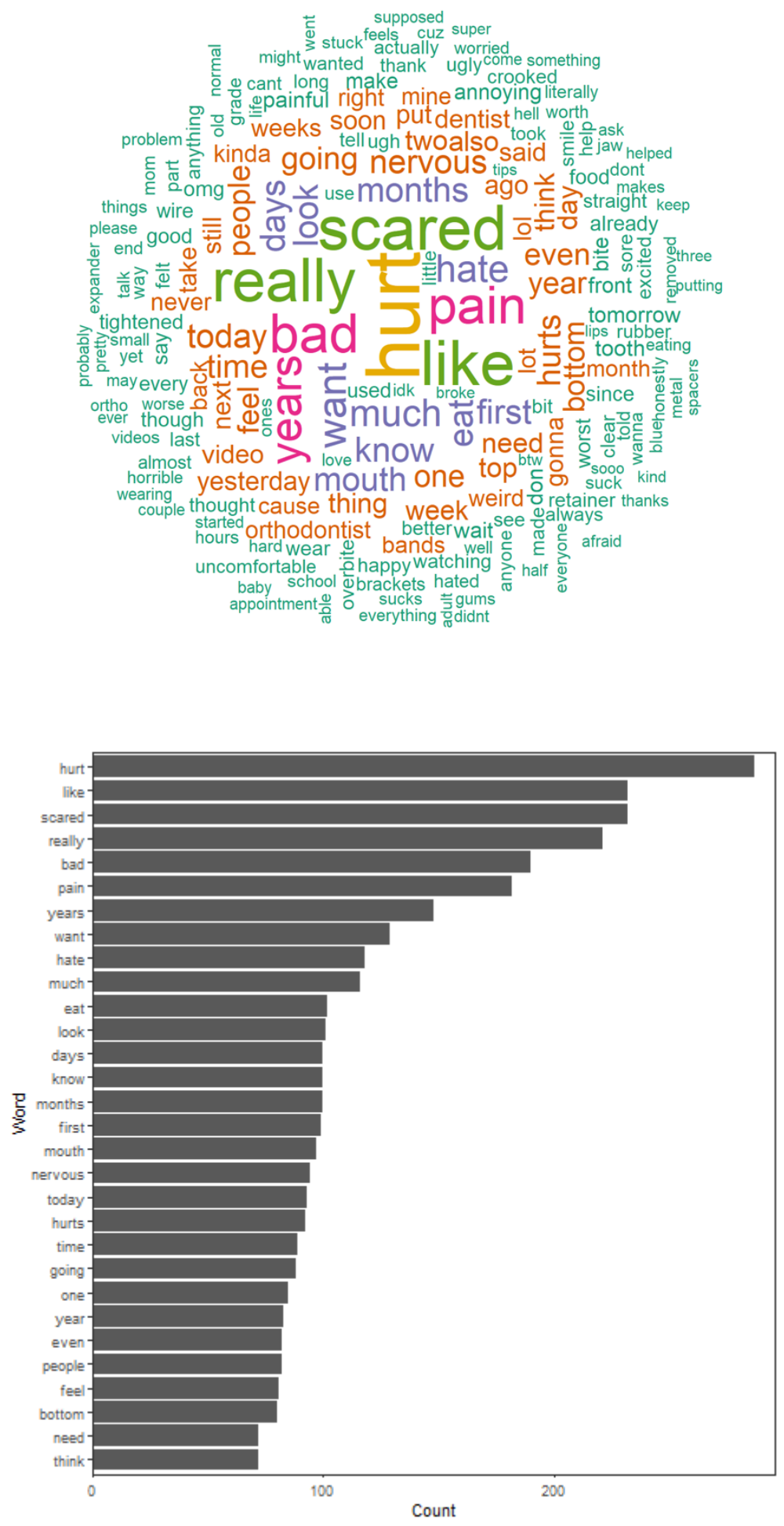
Figure 7. Most common words found in negative comments from IOA videos
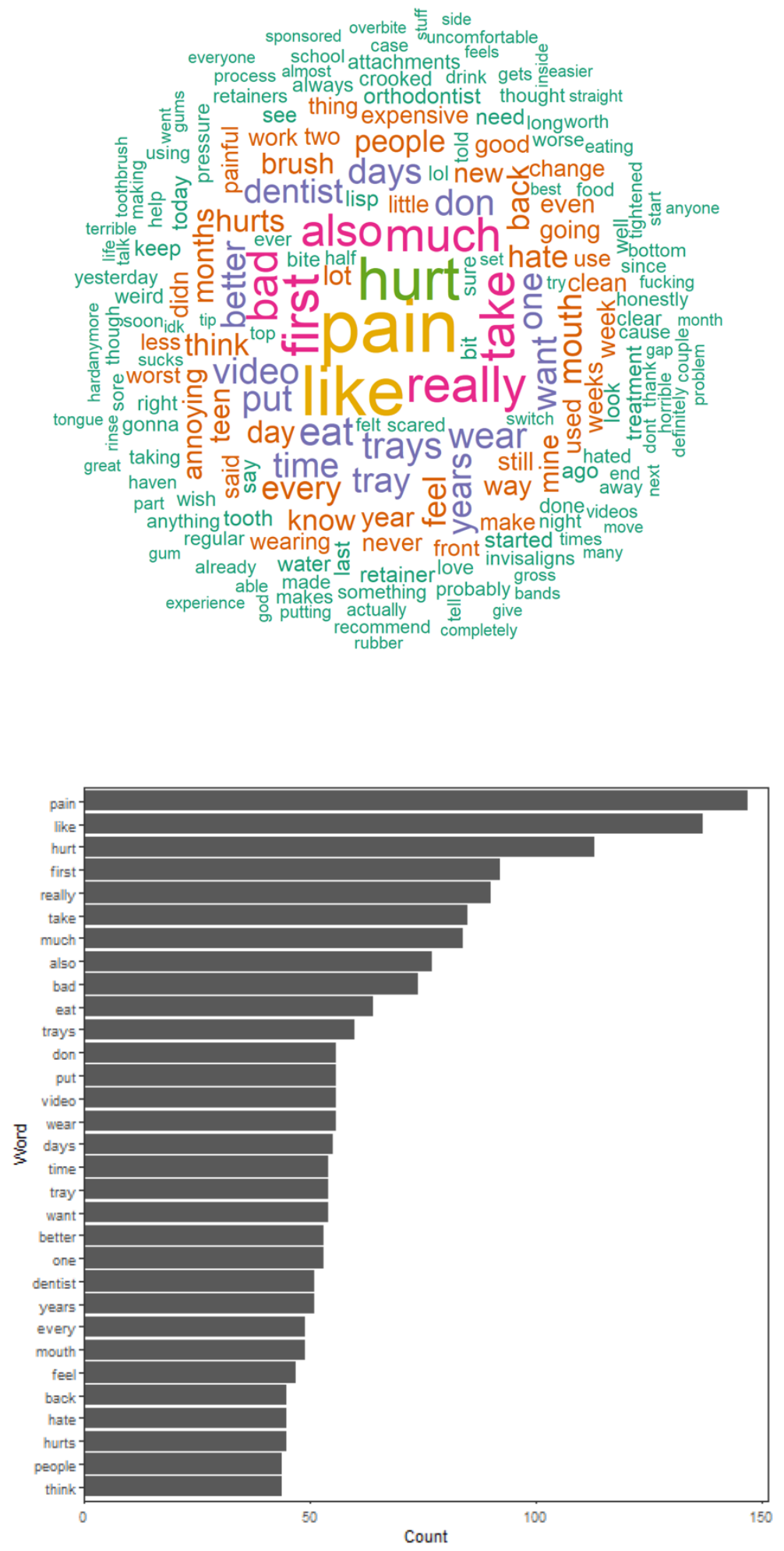
Figure 8. Most common words found in negative comments from DTCA videos
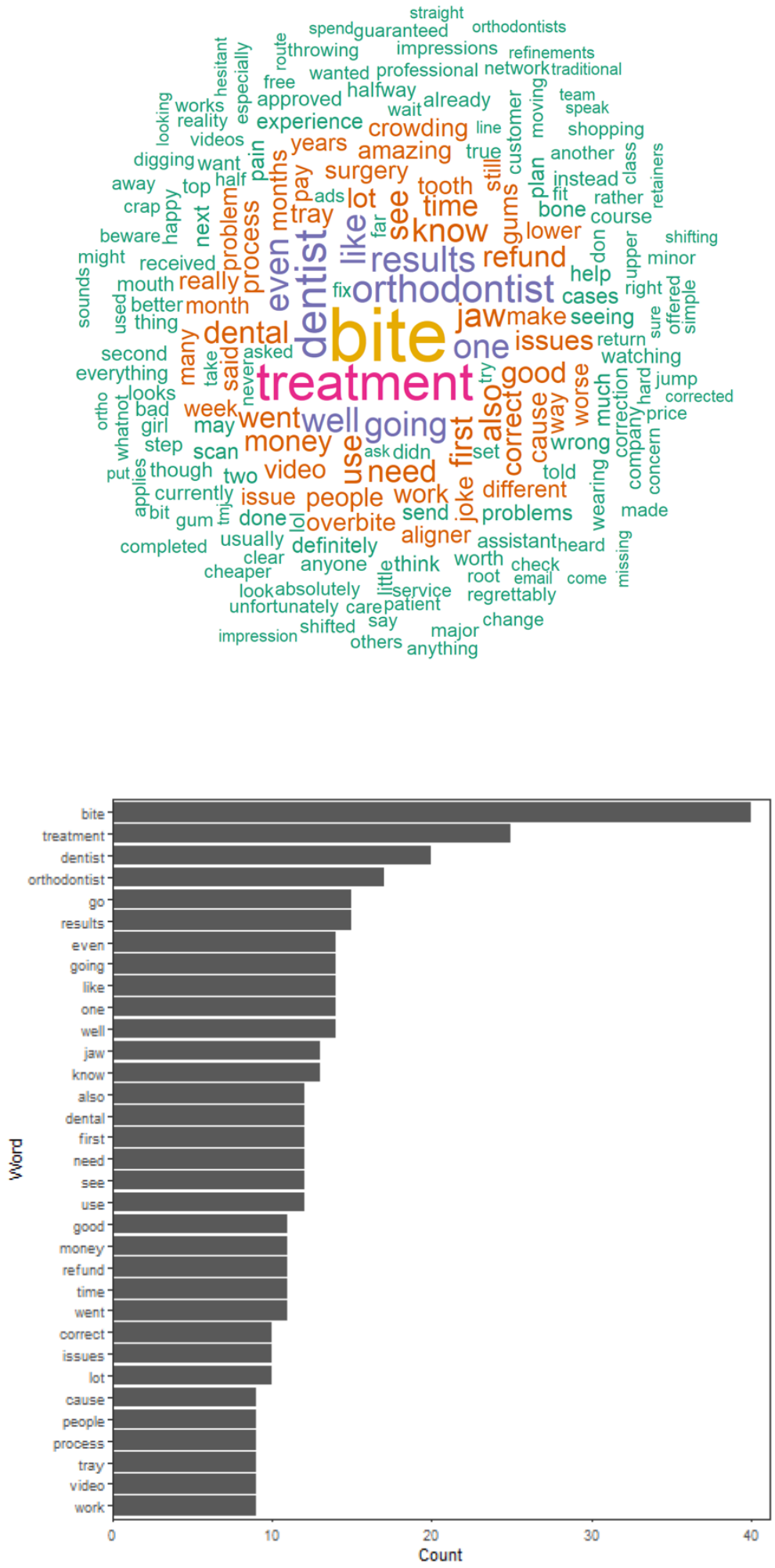